\title{
Efficacy and safety of adalimumab in Japanese patients with moderately to severely active ulcerative colitis
}

\author{
Yasuo Suzuki • Satoshi Motoya $\cdot$ Hiroyuki Hanai - Takayuki Matsumoto • \\ Toshifumi Hibi • Anne M. Robinson - Nael M. Mostafa • Jingdong Chao • \\ Vipin Arora $\cdot$ Anne Camez $\cdot$ Roopal B. Thakkar $\cdot$ Mamoru Watanabe
}

Received: 29 August 2013/Accepted: 26 November 2013/Published online: 24 December 2013

(C) The Author(s) 2013. This article is published with open access at Springerlink.com

\begin{abstract}
Background Adalimumab is a fully human, monoclonal antibody against tumor necrosis factor that is approved in Western countries for the treatment of moderately to severely active ulcerative colitis (UC).

Methods This 52-week, phase 2/3, randomized, doubleblind study evaluated adalimumab for induction and maintenance treatment in 273 anti-TNF-naive Japanese patients with UC who were refractory to corticosteroids, immunomodulators, or both. Patients received placebo, adalimumab $80 / 40$ ( $80 \mathrm{mg}$ at week 0 , then $40 \mathrm{mg}$ every other week), or adalimumab 160/80 (160/80 mg at weeks $0 / 2$, then $40 \mathrm{mg}$ every other week) in addition to background UC therapy.

Results At week 8, remission rates were similar among treatment arms, but more patients treated with adalimumab 160/80 achieved response (placebo, $35 \% ; 80 / 40,43 \%$; $160 / 80,50 \% ; P=0.044$ for $160 / 80$ vs placebo) and
\end{abstract}

Electronic supplementary material The online version of this article (doi:10.1007/s00535-013-0922-y) contains supplementary material, which is available to authorized users.

Y. Suzuki

Toho University Medical Center Sakura Hospital, Chiba, Japan

S. Motoya

IBD Center, Sapporo Kosei General Hospital, Sapporo, Japan

H. Hanai

Hamamatsu South Hospital, Shizuoka, Japan

T. Matsumoto

Hyogo College of Medicine, Hyogo, Japan

T. Hibi

Kitasato Institute Hospital, Tokyo, Japan mucosal healing (placebo, $30 \% ; 80 / 40,39 \% ; 160 / 80$, $44 \% ; P=0.045$ for $160 / 80$ vs placebo) compared with placebo. At week 52, more patients receiving adalimumab $40 \mathrm{mg}$ every other week achieved response (18 vs $31 \%$; $P=0.021)$, remission (7 vs $23 \% ; P=0.001)$, and mucosal healing (16 vs $29 \% ; P=0.015$ ) compared with placebo. Week 8 response to adalimumab was associated with greater rates of response (61\%), remission (46\%), and mucosal healing $(57 \%)$ at week 52 relative to the overall population. Rates of serious adverse events were similar between treatment arms.

Conclusions Induction with adalimumab 160/80 mg led to early response and mucosal healing. Maintenance adalimumab had greater rates of long-term response, remission, and mucosal healing compared with placebo. No new safety signals were identified.

Keywords Clinical remission - Japan - Ulcerative colitis $\cdot$ Mucosal healing $\cdot$ Adalimumab

\author{
A. M. Robinson - N. M. Mostafa - J. Chao - V. Arora · \\ R. B. Thakkar \\ AbbVie, North Chicago, IL, USA \\ A. Camez \\ AbbVie GmbH \& Co KG, Ludwigshafen, Germany \\ M. Watanabe $(\square)$ \\ Department of Gastroenterology, Tokyo Medical \\ and Dental University, 1-5-45 Yushima, Bunkyo-ku, \\ Tokyo 113-8519, Japan \\ e-mail: mamoru.gast@tmd.ac.jp
}




\section{Introduction}

Ulcerative colitis (UC) is a chronic inflammatory bowel disease with an unpredictable course of relapse and remission $[1,2]$. Inflammation and mucosal damage associated with UC are a consequence of immune dysregulation, including overexpression of proinflammatory cytokines such as tumor necrosis factor (TNF) [3]. Diseaserelated immune dysregulation has been associated with genetic polymorphisms, which may differ depending on the population being examined; for example, a recent metaanalysis found that inflammatory bowel disease susceptibility loci in Japanese patients are closely associated with human leukocyte antigen regions [4]. Japanese patients with UC who have a family history of the disease tend to have an earlier onset and a more severe course of disease [5].

Adalimumab is a subcutaneously administered, recombinant, fully human, monoclonal antibody directed against TNF. Phase III trials of adalimumab in Western patients with moderately to severely active UC who failed therapy with steroids and/or immunomodulators (including some patients who had previously failed other anti-TNF agents) demonstrated the ability of adalimumab to induce and maintain remission at an induction dose of $160 / 80 \mathrm{mg}$ (week 0/week 2) and a maintenance dose of $40 \mathrm{mg}$ every other week (EOW) [6-8]. Based on these results, adalimumab was approved in the United States and the European Union for the treatment of adult patients with moderately to severely active UC.

The incidence and prevalence of UC in Japan are lower than in Western countries, though they are rapidly increasing [9]. The safety and efficacy of adalimumab in the treatment of Crohn's disease (CD) in Japanese patients have been recently demonstrated [10]. Here, we report the results of a 52-week efficacy and safety trial of adalimumab in anti-TNF-naïve Japanese patients with moderately to severely active UC.

\section{Methods}

\section{Study design}

This 52-week, phase II/III, randomized, double-blind, placebo-controlled study evaluated the efficacy, safety, and pharmacokinetics of adalimumab for induction and maintenance therapy in Japanese patients with moderately to severely active UC. The objective of this study was to show the directional similarity of study results compared with Western studies [6-8] using descriptive statistics. The study was conducted at 65 centers in Japan between February 2009 and May 2011. The protocol was approved by the institutional review board of each center, and each patient provided written consent. For patients aged 19 and younger, written consent was also provided by the patient's guardian.

\section{Patient population}

Japanese patients $\geq 15$ years of age with biopsy-confirmed, moderately to severely active UC (Mayo score [11] [a composite of stool frequency, rectal bleeding, physician's global assessment (PGA), and endoscopy subscores] of 6-12 points and an endoscopy subscore of $\geq 2$ ) despite concurrent treatment with stable doses of oral corticosteroids (prednisolone equivalent of $\geq 20 \mathrm{mg} / \mathrm{day}$ for $\geq 2$ weeks or 5 to $<20 \mathrm{mg} /$ day for $\geq 40$ days before baseline) and/or immunomodulators (azathioprine $\geq 50 \mathrm{mg} /$ day or 6-mercaptopurine [6-MP] $\geq 30 \mathrm{mg} /$ day for at least 90 days) were enrolled. If patients were receiving both oral corticosteroids and immunomodulators at baseline, only one of the drugs needed to meet the above criteria. Patients who were previously treated with corticosteroids or immunomodulators during the past 5 years and who, in the judgment of the investigator, had failed to respond or who could not tolerate their treatment were also eligible for enrollment. Patients who had received prior treatment with anti-TNF therapies or other biologic agents were not eligible.

Key exclusion criteria included the following: planned bowel surgery; discontinuation of oral corticosteroids within 2 weeks before baseline; receipt of corticosteroid injection, cyclosporine, tacrolimus, or mycophenolate mofetil within 4 weeks before baseline; receipt of therapeutic enema or suppository, other than required for endoscopy, within 2 weeks before screening endoscopy; receipt of cytapheresis within 56 days of baseline; receipt of total parenteral nutrition during the screening period; receipt of any investigational agent within 4 weeks or 5 half-lives before baseline; or nonstable doses or recent (within 4 weeks) discontinuation of oral aminosalicylates or UC-related antibiotics (e.g., ciprofloxacin, metronidazole). Other reasons for exclusion included the following: current diagnosis of fulminant colitis or toxic megacolon; disease limited to the rectum (ulcerative proctitis); current diagnosis of indeterminate colitis or $\mathrm{CD}$; positive Clostridium difficile toxin stool assay result; recent history of infection requiring antimicrobial therapy; evidence of tuberculosis infection by chest X-ray or purified protein derivative skin test; administration of a live vaccine within 90 days before baseline; history of listeria or histoplasmosis, active tuberculosis, human immunodeficiency virus, immunodeficiency syndrome, central nervous system demyelinating disease, or malignancy (except successfully treated, nonmetastatic, cutaneous 
squamous cell or basal cell carcinoma, or localized carcinoma in situ of the cervix); evidence of colonic dysplasia; history of poorly controlled medical conditions (e.g., uncontrolled diabetes); or known hypersensitivity to excipients of adalimumab.

\section{Treatment administration}

Patients were randomly assigned in a 1:1:1 ratio to subcutaneous injections of adalimumab $(160 \mathrm{mg}$ at week 0 , $80 \mathrm{mg}$ at week 2, and then $40 \mathrm{mg}$ EOW beginning at week 4 [160/80 arm], or $80 \mathrm{mg}$ at week $0,40 \mathrm{mg}$ at week 2, and then $40 \mathrm{mg}$ EOW beginning at week 4 [80/40 arm]), or placebo administered by a physician or nurse. The study drug was added to ongoing background therapy for UC (no therapies were washed out). Randomization was based on a centrally designed randomization table. Changes in doses of UC-related concomitant medications other than corticosteroids were not permitted during the study. After the initial 8-week induction period, patients who responded to treatment according to the judgment of the investigator were allowed to taper their corticosteroid dose. Patients with an inadequate response to the study drug (partial Mayo score greater than or equal to that of the baseline score on two consecutive visits at least 14 days apart for patients with a baseline partial Mayo score of 3-7, or partial Mayo score $\geq 7$ on two consecutive visits at least 14 days apart for patients with a baseline partial Mayo score of 8 or 9) or with a flare (partial Mayo score increase of $\geq 3$ compared with the score at the last evaluation before the disease flare on two consecutive visits at least 14 days apart) at or after week 8 were entered into the "rescue arm." Treatment in the "rescue arm" consisted of 4 weeks of blinded adalimumab (either $160 \mathrm{mg}$ initially and $80 \mathrm{mg}$ 2 weeks later for patients in the placebo arm, or $40 \mathrm{mg}$ initially and 2 weeks later for patients in either adalimumab arm) followed by open-label adalimumab $40 \mathrm{mg}$ EOW, with a possibility to escalate to $80 \mathrm{mg}$ EOW in case of inadequate response or disease flare at least 8 weeks later.

\section{Efficacy evaluations}

The last evaluation before the first dose of the study drug was used as baseline for all analyses. Full Mayo scores and Inflammatory Bowel Disease Questionnaire (IBDQ, used under license from McMaster University [12]) scores were determined at weeks 8, 32, and 52. The Mayo subscores for stool frequency and rectal bleeding were calculated based on entries from patient diaries using the worst diary entry from the 3 days before each study visit for each subscore. Partial Mayo score (Mayo score excluding the endoscopy subscore) was evaluated EOW during the first 8 weeks and monthly thereafter. Efficacy endpoints analyzed included response per full Mayo score (decrease of $\geq 3$ points and $\geq 30 \%$ from baseline plus a decrease in the rectal bleeding subscore [RBS] $\geq 1$ or an absolute RBS of $\leq 1$ ), remission (full Mayo score $\leq 2$ with no individual subscore $>1$ ), and mucosal healing (endoscopy subscore $\leq 1$ ) at weeks 8,32 , and 52 .

Other efficacy analyses at weeks 8,32 , and 52 included RBS, PGA, and stool frequency indicative of mild disease (score $\leq 1)$ and IBDQ response ( $\geq 16$-point increase from baseline in IBDQ score). In addition, the study evaluated the response per partial Mayo score (decrease of $\geq 2$ points and $\geq 30 \%$ from baseline plus a decrease in the RBS $\geq 1$ or an absolute RBS of $\leq 1$ ) from weeks 2 to 8 and rates of steroid-free status and steroid-free remission at week 32 and week 52 in the subset of patients taking corticosteroids at baseline. Subgroup analyses included the proportion of patients with remission at week 8 and at week 52 by baseline concomitant medication use (corticosteroids and immunomodulators), and week 52 efficacy (response, remission, and mucosal healing) in patients who achieved response per full and partial Mayo score at week 8. Other analyses included comparison of remission rates at week 8 using full Mayo scores calculated with different calculation methods for the rectal bleeding and stool frequency subscores (worst vs. mean diary entries and 5 vs. 3 days of diary entries), in order to explore the influence of subscore calculation methodology on efficacy rates. Currently, there is not a standard method for the determination of these subscores in terms of days of diary entries used or the use of mean or worst rank approaches, and different studies have used different approaches. For example, the Western adalimumab studies used the same method that this study used (worst entry of 3 days of diary entries), whereas the golimumab UC development program used the average of 3 days of diary entries [13].

\section{Pharmacokinetics and immunogenicity}

Blood samples for serum adalimumab concentrations were obtained at baseline and weeks 2, 4, 8, 32, and 52. Samples for serum anti-adalimumab antibodies (AAAs) were obtained at baseline and weeks 8, 32, and 52. All blood samples for serum adalimumab and AAA concentrations were obtained before scheduled drug injections. Serum adalimumab concentrations were determined in a central laboratory using a validated enzyme-linked immunosorbent assay method, which was adapted from Weisman et al. [14] using diluted samples. Serum AAA concentrations were determined using a validated double-antigen immunoassay that detects antibodies directed against epitopes on the entire adalimumab molecule. Similar to other immunoassays available at the time of the performance of the study, the assay detects only free (unbound) AAA. Because the 
presence of adalimumab in the sample competes with the capture and detector adalimumab for binding to the AAAs, serum samples with an adalimumab concentration $>2 \mu \mathrm{g} /$ $\mathrm{mL}$ were not analyzed for AAAs. Subjects were considered to have AAAs if they had at least one AAA-positive sample.

\section{Safety assessments}

Patients were monitored continuously for adverse events (AEs), including evaluations every 2 weeks from week 0 to week 52. Other safety parameters (vital signs and clinical laboratory parameters) were ascertained monthly.

\section{Statistical methods}

\section{Sample size}

The target sample size for this study was 85 per arm (total subjects: 225). The sample size was based on expert opinion, taking into consideration the clinical remission rate of adalimumab and infliximab in Western subjects with CD. The sample size was smaller than in Western studies due to the lower prevalence of UC in Japan [9] compared with Western countries. This trial was designed to allow comparison of directionality of effect with previously published Western studies [6,7]. At week 8 , the expected remission rates were $30 \%$ in the 160/80 arm, $22 \%$ in the 80/40 arm, and $15 \%$ in the placebo arm. At week 52, the expected rates were $12 \%$ in the combined adalimumab arm $(160 / 80+80 / 40)$ and $7 \%$ in the placebo arm. The expected probability that week 8 remission would be highest in the 160/80 arm and lowest in the placebo arm was greater than $80 \%$; likewise, there was also a greater than $80 \%$ expected probability that the week 52 remission rate would be higher in the combined adalimumab arm than in the placebo arm.

\section{Efficacy analyses}

All statistical analyses were exploratory. The efficacy and safety analyses were conducted on a full analysis set (FAS) that included all patients who received $\geq 1$ dose of the double-blind study drug. Nonresponder imputation, whereby the patient was assumed to not have efficacy, was used for patients with missing data or those who moved to the rescue arm for all efficacy endpoints.

Efficacy analyses for categorical endpoints were performed using the chi-squared test for adalimumab vs. placebo. For week 8 and earlier endpoints, data were analyzed by three induction arms $(160 / 80,80 / 40$, or placebo). Because both adalimumab induction dosing groups received the same maintenance dose of $40 \mathrm{mg}$ EOW, two arms were analyzed (combined adalimumab or placebo) for time points after week 8 .

\section{Safety analyses}

Adverse events and mean changes from baseline in laboratory variables and vital signs occurring during doubleblind therapy were summarized within the FAS. Adverse events in adalimumab-treated patients with response per full Mayo score at week 8 were also evaluated.

\section{Pharmacokinetic analyses}

Adalimumab concentrations were summarized at each time point using descriptive statistics. The data from all patients randomized to adalimumab who received $\geq 1$ dose of adalimumab and had $\geq 1$ measurable serum adalimumab concentration were included in the pharmacokinetic analyses.

\section{Results}

Disposition and demographics

A total of 343 patients provided informed consent, and 274 were randomized. Of these 274 patients, 273 (96 in the placebo arm, 87 in the $80 / 40$ arm, and 90 in the 160/80 arm) constituted the FAS (Fig. 1).

The one patient not included in the FAS inadvertently received rescue medication instead of double-blind treatment and was discontinued from the study.

Study participants were predominantly male and representative of a moderately to severely active UC population, with a mean duration of UC of approximately 8 years and a baseline Mayo score of approximately 9 (Table 1). More than $60 \%$ of patients in each arm had pancolitis, with the highest percentage of pancolitis $(70 \%)$ occurring in the adalimumab 160/80 arm. The majority of patients were taking UC-related medications at baseline, with $\geq 90 \%$ receiving aminosalicylic acids, $\geq 60 \%$ taking steroids, and $\geq 40 \%$ receiving immunomodulators. The highest rate of baseline steroid use was in the adalimumab 80/40 arm and the highest rate of immunomodulator use was in the placebo arm.

During 52 weeks of treatment, more patients in the placebo arm (66\%) moved to rescue therapy than in the adalimumab $80 / 40(57 \%)$ or $160 / 80(51 \%)$ arms. The rate of study completion on double-blind therapy was higher in the adalimumab 160/80 arm (40\%) than the placebo (27\%) or $80 / 40(33 \%)$ arms (Fig. 1). 


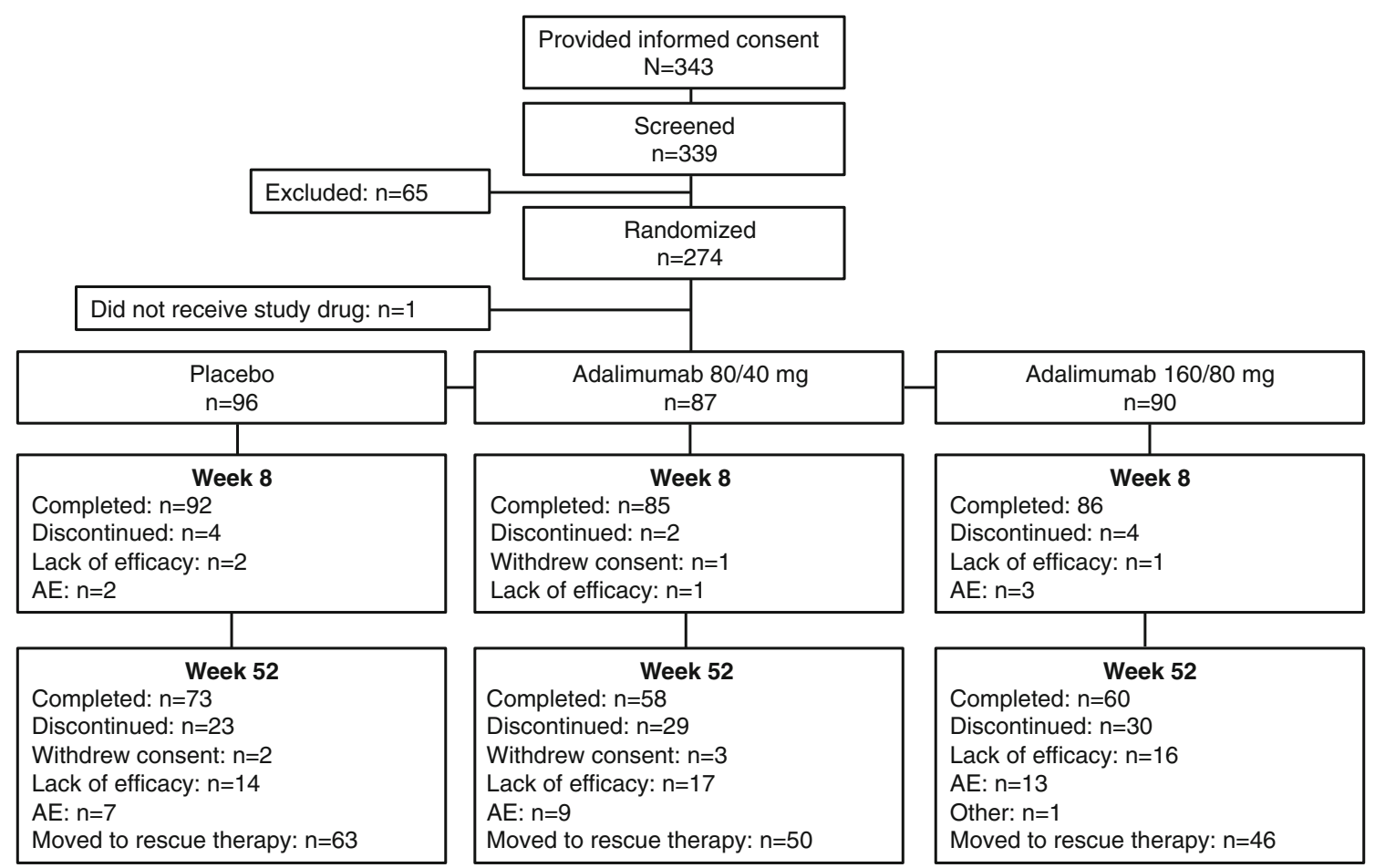

Fig. 1 Patient disposition. $A E$ adverse event

\section{Efficacy}

\section{Induction therapy}

The rates of early response (per partial Mayo score at weeks 2, 4, and 6, and per full Mayo score at week 8) are shown in Fig. 2a. Adalimumab 160/80 mg treatment was associated with the highest rates of clinical response at each time point $(P=0.044$ at week 8 vs. placebo). Week 8 remission and mucosal healing rates by treatment arm are shown in Fig. $2 b$. Remission rates were similar between placebo and both adalimumab treatment arms, but adalimumab 160/80 mg was associated with the highest rate of mucosal healing ( $P=0.045$ for $160 / 80 \mathrm{mg}$ vs placebo). The proportions of patients with improvement in each Mayo subscore (reduction by $\geq 1$ ) and IBDQ response were greater in the adalimumab arms compared with placebo (Table 2).

\section{Maintenance therapy}

Maintenance therapy with adalimumab $40 \mathrm{mg}$ EOW was associated with higher rates of response, remission, and mucosal healing at weeks 32 and 52 compared with placebo (Fig. 3). Rates of remission for the adalimumabtreated patients were greater than placebo at both weeks 32 and $52(P=0.038$ and $P=0.001$, respectively; Fig. $3 b)$. The proportion of adalimumab-treated patients with remission increased over time, with the highest rate $(23.2 \%)$ observed at week 52 in spite of the use of a nonresponder imputation analysis. Rates of response (Fig. 3a) and mucosal healing (Fig. 3c) were sustained compared with placebo over time.

The percentage of patients receiving corticosteroids at baseline who were steroid-free and the percentages who achieved steroid-free remission during the maintenance period are shown in Table 2 . Nearly one-third $(32.5 \%)$ of adalimumab-treated patients taking steroids at baseline were steroid-free at week 52 compared with $20.7 \%$ of patients taking placebo.

\section{Adalimumab week 8 responders}

Patients exhibiting response to adalimumab induction therapy at week 8 were more likely to achieve efficacy at week 52 compared with the overall adalimumab-treated population. Among the 82 patients who achieved response per full Mayo score at week 8, $61 \%$ maintained response at week 52, $46 \%$ were in remission, and $57 \%$ had mucosal healing. Similar values were observed when week 8 response was determined using partial Mayo score $(N=81): 58 \%$ for response, $44 \%$ for remission, and $54 \%$ for mucosal healing.

\section{Efficacy by baseline concomitant medications}

At week 8 , there was a high rate of remission noted in patients in the placebo arm who were not receiving immunomodulators $(22.7 \%)$ and those receiving corticosteroids (17.2 \%; 
Table 1 Patient demographics and baseline characteristics (FAS)

\begin{tabular}{|c|c|c|c|}
\hline Characteristic & Placebo $(N=96)$ & ADA $80 / 40 \mathrm{mg}(N=87)$ & ADA $160 / 80 \mathrm{mg}(N=90)$ \\
\hline Male, $n(\%)$ & $70(72.9)$ & $50(57.5)$ & $61(67.8)$ \\
\hline Age, mean \pm SD (years) & $41.3 \pm 13.6$ & $44.4 \pm 15.0$ & $42.5 \pm 14.6$ \\
\hline Weight, mean \pm SD $(\mathrm{kg})$ & $60.8 \pm 14.1$ & $58.7 \pm 11.1$ & $60.1 \pm 12.3$ \\
\hline Tobacco, nonsmoker, $n(\%)$ & $55(57.3)$ & $53(60.9)$ & $50(55.6)$ \\
\hline Alcohol, nondrinker, $n(\%)$ & $36(37.5)$ & $45(51.7)$ & $43(47.8)$ \\
\hline \multicolumn{4}{|l|}{ Duration of UC (years) } \\
\hline Mean \pm SD & $7.8 \pm 6.6$ & $8.3 \pm 7.7$ & $7.8 \pm 7.1$ \\
\hline Range & $0.6-26.6$ & $0.8-37.8$ & $0.4-32.5$ \\
\hline \multicolumn{4}{|l|}{ Site of UC, $n(\%)$} \\
\hline Pancolitis & $59(61.5)$ & $54(62.1)$ & $63(70.0)$ \\
\hline Descending colon & $35(36.5)$ & $32(36.8)$ & $27(30.0)$ \\
\hline Other $^{\mathrm{a}}$ & $2(2.1)$ & $1(1.1)$ & 0 \\
\hline Mayo score, mean \pm SD & $8.5 \pm 1.6$ & $8.5 \pm 1.4$ & $8.6 \pm 1.4$ \\
\hline Partial Mayo score, mean \pm SD & $6.1 \pm 1.3$ & $6.0 \pm 1.3$ & $6.2 \pm 1.4$ \\
\hline IBDQ score, mean $\pm \mathrm{SD}^{\mathrm{b}}$ & $148.2 \pm 28.9$ & $144.9 \pm 28.7$ & $146.0 \pm 31.7$ \\
\hline C-reactive protein, median (range) $(\mathrm{mg} / \mathrm{dL})$ & $0.34(0.05-8.72)$ & $0.31(0.05-10.77)$ & $0.22(0.05-6.28)$ \\
\hline \multicolumn{4}{|l|}{ Baseline UC medication, $n(\%)$} \\
\hline 5-ASAs & $89(92.7)$ & $84(96.6)$ & $83(92.2)$ \\
\hline Immunomodulators (AZA, 6-MP) & $52(54.2)$ & $38(43.7)$ & $41(45.6)$ \\
\hline Systemic corticosteroids & $58(60.4)$ & $63(72.4)$ & $57(63.3)$ \\
\hline
\end{tabular}

Baseline is the last measurement time point before the first dose of study medication in the induction period

$A D A$ adalimumab, 5-ASA 5-aminosalicylic acid, AZA azathioprine, $F A S$ full analysis set, $I B D Q$ Inflammatory Bowel Disease Questionnaire, 6$M P$ 6-mercaptopurine, $S D$ standard deviation, $U C$ ulcerative colitis

a Two patients (distal colitis and appendix/ascending colon/rectal colon/transverse colon) in the placebo arm and one patient (rectal to sigmoidal colon) in the 80/40 mg arm

b Placebo, $n=96 ; 80 / 40$ arm, $n=85 ; 160 / 80$ arm, $n=88$

Supplementary Table S1). Baseline steroids or immunomodulators had an inconsistent effect in the adalimumab arms, depending on the dose. At week 52, there was a notable negative effect of baseline steroid use on remission rates. Patients in the placebo arm who were not receiving immunomodulators at baseline had a higher rate of remission at week 52 than those who were taking them; the opposite effect was noted in the adalimumab-treated patients.

\section{Effect of Mayo subscore calculation method on remission rates}

Rates of remission at week 8 for all three arms and the treatment effect sizes (adalimumab rate minus placebo rate) were affected by the method used to assign values at the study visit for Mayo score subscores for stool frequency and rectal bleeding (Supplementary Table S2). The lowest rates of remission and the smallest effect sizes were observed when subscores were based on the worst daily patient diary entries during a 5-day or a 3-day period. Remission rates and effect sizes were greater when the Mayo scores were based on the average of these two subscores from 3 or 5 days of diary entries, with the mean of the 5-day method resulting in the highest overall remission rates and the largest effect sizes.

\section{Pharmacokinetics/immunogenicity}

Adalimumab trough serum concentrations by randomized group and by remission status at week 8 and week 52 are shown in Table 3 . No adalimumab was detected in any samples at week 0 . Within each dosing arm, serum concentrations were similar by remission status at week 8 . At week 52 , patients with remission had slightly higher serum adalimumab concentrations than those who did not achieve remission, with significant overlap by remission status. Overall, 12 of $240(5.0 \%)$ patients who received adalimumab during the study developed AAAs.

\section{Safety}

The overall safety profile of adalimumab was similar to that observed in other clinical trials, including trials in patients with UC [6-8] and CD [10, 15, 16], and no new 

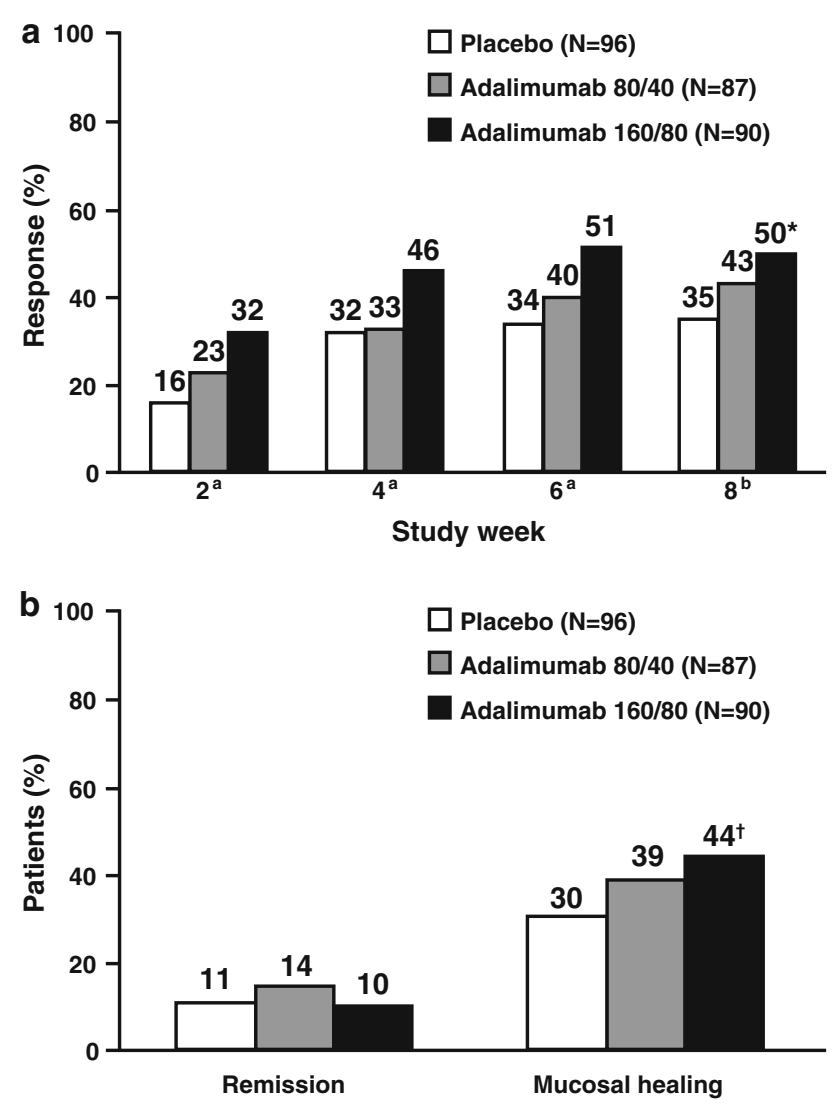

Fig. 2 Response during induction therapy (a) and remission and mucosal healing at week 8 (b; FAS, NRI). FAS full analysis set, NRI nonresponder imputation. ${ }^{\mathrm{a}}$ Per partial Mayo score. ${ }^{\mathrm{b}}$ Per full Mayo score. ${ }^{*} P=0.044 .{ }^{\dagger} P=0.045$ safety signals were observed. Most AEs were nonserious, mild, or moderate in severity, and were considered "not related" or "probably not related" to the study drug by the investigator. During the induction period, the overall rate of AEs was similar in the placebo arm vs. the 80/40 and 160/80 arms (Table 4). Serious AEs and worsening or flare of UC were most common in the placebo arm, and infections and injection site reactions were most common in the adalimumab 160/80 arm. There were three serious infections during the induction period, all of which occurred in the adalimumab 160/80 arm. Exposure-adjusted rates of AEs occurring during 52 weeks of double-blind treatment for patients receiving placebo, patients receiving adalimumab, and patients with response to adalimumab at week 8 per full Mayo score are shown in Table 4. Rates for overall AEs and serious AEs were similar between placebo and adalimumab. Patients receiving adalimumab were more likely to experience AEs considered to be at least possibly drug-related, AEs leading to discontinuation, serious infections, and injection site reactions compared to patients receiving placebo. All injection site reactions were mild, and most were managed without study drug interruption or discontinuation. Patients receiving placebo were more likely to experience worsening or flare of UC. Compared with the overall adalimumab population, patients with response at week 8 were less likely to experience an $\mathrm{AE}$, serious $\mathrm{AE}$, injection site reaction, or flare/ worsening of UC.

During the entire trial, two malignancies were reported, both of which were considered to be unrelated to the study

Table 2 Other efficacy endpoints (FAS, NRI)

\begin{tabular}{|c|c|c|c|c|c|c|c|}
\hline \multirow[t]{2}{*}{ Endpoint, $n(\%)^{\mathrm{a}}$} & \multicolumn{3}{|l|}{ Week 8} & \multicolumn{2}{|l|}{ Week 32} & \multicolumn{2}{|l|}{ Week 52} \\
\hline & $\begin{array}{l}\text { Placebo } \\
(N=96)\end{array}$ & $\begin{array}{l}\text { ADA } 80 / 40 \mathrm{mg} \\
(N=87)\end{array}$ & $\begin{array}{l}\text { ADA } 160 / 80 \mathrm{mg} \\
(N=90)\end{array}$ & $\begin{array}{l}\text { Placebo } \\
(N=96)\end{array}$ & $\begin{array}{l}\text { ADA } 40 \mathrm{mg} \text { EOW } \\
(N=177)\end{array}$ & $\begin{array}{l}\text { Placebo } \\
(N=96)\end{array}$ & $\begin{array}{l}\text { ADA } 40 \mathrm{mg} \text { EOW } \\
(N=177)\end{array}$ \\
\hline $\begin{array}{l}\text { Rectal bleeding } \\
\text { subscore } \leq 1\end{array}$ & $65(67.7)$ & $70(80.5)^{*}$ & $64(71.1)$ & $27(28.1)$ & $74(41.8)$ & $22(22.9)$ & $59(33.3)$ \\
\hline PGA subscore $\leq 1$ & $43(44.8)$ & $41(47.1)$ & $55(61.1)^{*}$ & $27(28.1)$ & $66(37.3)$ & $19(19.8)$ & $57(32.2)^{*}$ \\
\hline $\begin{array}{l}\text { Stool frequency } \\
\text { subscore } \leq 1\end{array}$ & $31(32.3)$ & $30(34.5)$ & $36(40.0)$ & $20(20.8)$ & $57(32.2)^{*}$ & $13(13.5)$ & $51(28.8)^{*}$ \\
\hline IBDQ response ${ }^{\mathrm{b}}$ & $38(39.6)$ & $42(48.3)$ & $38(42.2)$ & $21(21.9)$ & $55(31.1)$ & $12(12.5)$ & $45(25.4)^{\dagger}$ \\
\hline Steroid-free ${ }^{c}$ & ND & ND & ND & $12(20.7)$ & $35(29.2)$ & $12(20.7)$ & $39(32.5)$ \\
\hline $\begin{array}{l}\text { Steroid-free } \\
\text { remission }^{\mathrm{c}}\end{array}$ & ND & ND & ND & $5(8.6)$ & $12(10.0)$ & $4(6.9)$ & $17(14.2)$ \\
\hline
\end{tabular}

$A D A$ adalimumab, EOW every other week, $F A S$ full analysis set, $I B D Q$ Inflammatory Bowel Disease Questionnaire, $N D$ not determined, $N R I$ nonresponder imputation, $P G A$ physician's global assessment, $U C$ ulcerative colitis

a Unless otherwise noted

${ }^{\mathrm{b}}$ Response was defined as an increase in IBDQ score of $\geq 16$ points from baseline

${ }^{\mathrm{c}}$ Percentages calculated based on the number of patients taking steroids at baseline (placebo, $N=58$; ADA $40 \mathrm{mg}$ EOW, $N=120$ )

* $P \leq 0.05,{ }^{\dagger} P \leq 0.01$ 


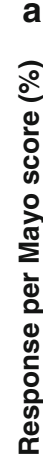
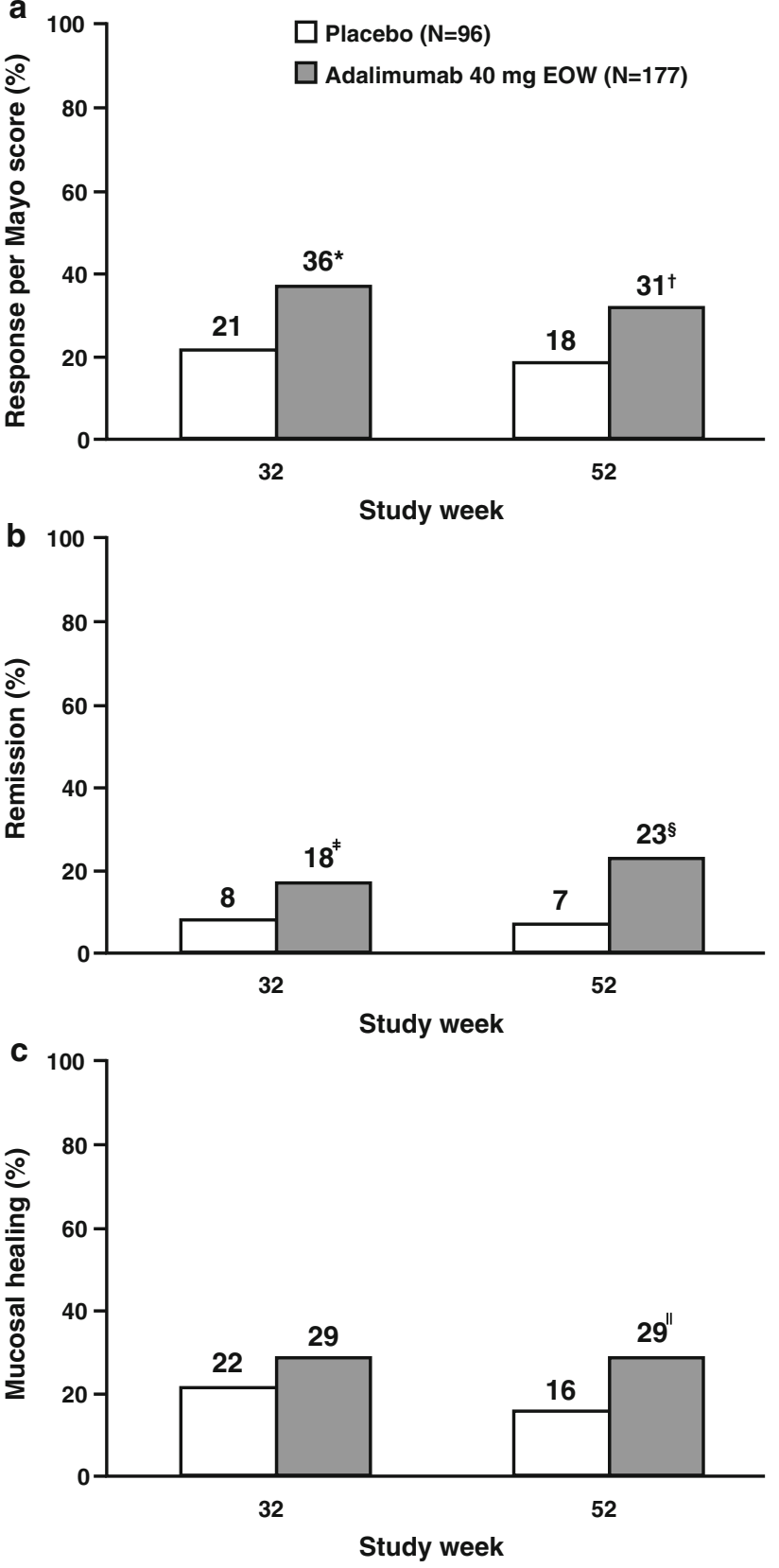

Fig. 3 Response (a), remission (b), and mucosal healing (c) during maintenance therapy (FAS, NRI). EOW every other week, $F A S$ full analysis set, $N R I$ nonresponder imputation. ${ }^{*} P=0.011 .{ }^{\dagger} P=0.021$. ${ }^{\star} P=0.038 .{ }^{\circledR} P=0.001 .{ }^{\|} P=0.015$

drug by the investigator. One case of tuberculosis was reported on day 73 (44 days after the last dose of the study drug) in a 65-year-old patient in the adalimumab 160/80 arm receiving prednisone $20 \mathrm{mg} / \mathrm{day}$; this patient had presented with a negative purified protein derivative test (erythema $>10 \mathrm{~mm}$ but no induration) and chest X-ray at baseline. The patient was hospitalized on day 81 and died on day 91 . There were no cases of lymphoma, melanoma, congestive heart failure, demyelination, or lupus-like syndrome.

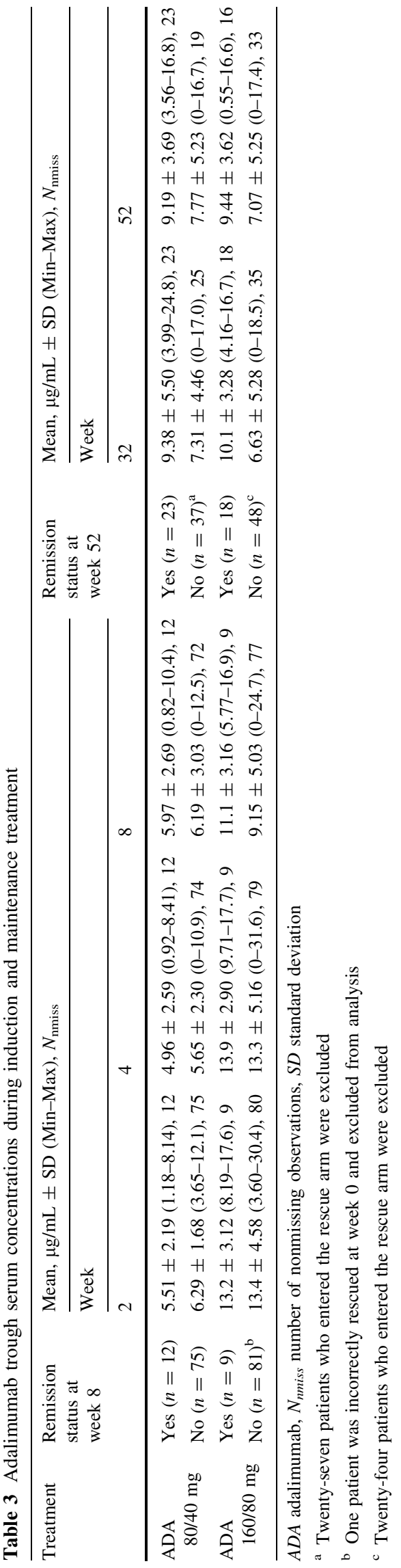


Table 4 Overview of adverse events occurring during double-blind therapy

\begin{tabular}{|c|c|c|c|c|c|c|}
\hline \multirow[t]{2}{*}{$\mathrm{AE}, n(\%)$} & \multicolumn{3}{|c|}{ Week $8, n(\%)$} & \multicolumn{3}{|c|}{ Week 52, E (E/100 PY) } \\
\hline & $\begin{array}{l}\text { Placebo } \\
(N=96)\end{array}$ & $\begin{array}{l}\text { ADA } \\
80 / 40 \mathrm{mg} \\
(N=87)\end{array}$ & $\begin{array}{l}\text { ADA } \\
160 / 80 \mathrm{mg} \\
(N=90)\end{array}$ & $\begin{array}{l}\text { Placebo } \\
(N=96) \\
\mathrm{PY}=44.8\end{array}$ & $\begin{array}{l}\text { ADA } 40 \mathrm{mg} \text { EOW } \\
(N=177) \\
\mathrm{PY}=98.2\end{array}$ & $\begin{array}{l}\text { ADA week } 8 \text { responders } \\
\text { per full Mayo score } \\
(N=82) \text { PY }=68.7\end{array}$ \\
\hline Any AE & $45(46.9)$ & $49(56.3)$ & $40(44.4)$ & $273(609.4)$ & $538(547.9)$ & $343(499.3)$ \\
\hline $\begin{array}{l}\text { At least possibly } \\
\text { drug-related }\end{array}$ & $10(10.4)$ & $14(16.1)$ & $12(13.3)$ & $34(75.9)$ & $91(92.7)$ & $64(93.2)$ \\
\hline Serious & $7(7.3)$ & $2(2.3)$ & $4(4.4)$ & $14(31.3)$ & $33(33.6)$ & $20(29.1)$ \\
\hline $\begin{array}{l}\text { Leading to early } \\
\text { discontinuation }\end{array}$ & $4(4.2)$ & 0 & $6(6.7)$ & $6(13.4)$ & $22(22.4)$ & $11(16.0)$ \\
\hline \multicolumn{7}{|l|}{$\mathrm{AE}$ of interest } \\
\hline Infection & $15(15.6)$ & $11(12.6)$ & 17 (18.9) & $70(156.3)$ & $134(136.5)$ & $90(131.0)$ \\
\hline Serious infection & 0 & 0 & $3(3.3)$ & $2(4.5)$ & $8(8.1)$ & $6(8.7)$ \\
\hline Malignancy $^{\mathrm{a}}$ & 0 & 0 & $1(1.1)$ & 0 & $2(2.0)$ & $1(1.5)$ \\
\hline Injection site reaction & $2(2.1)$ & $5(5.7)$ & $7(7.8)$ & $4(8.9)$ & $20(20.4)$ & $9(13.1)$ \\
\hline $\begin{array}{l}\text { Opportunistic infection } \\
\text { (excluding tuberculosis) }^{\mathrm{b}}\end{array}$ & 0 & 0 & $1(1.1)$ & 0 & $2(2.0)$ & $2(2.9)$ \\
\hline Tuberculosis & 0 & 0 & $1(1.1)$ & 0 & $1(1.0)$ & 0 \\
\hline Hepatic event & $1(1.0)$ & 0 & $1(1.1)$ & $3(6.7)$ & $5(5.1)$ & $3(4.4)$ \\
\hline Allergic reaction & 0 & $1(1.1)$ & 0 & $2(4.5)$ & $6(6.1)$ & $5(7.3)$ \\
\hline Hematologic event & $1(1.0)$ & $3(3.4)$ & $1(1.1)$ & $4(8.9)$ & $6(6.1)$ & $4(5.8)$ \\
\hline UC worsening/flare & $8(8.3)$ & $2(2.3)$ & $2(2.2)$ & 15 (33.5) & 18 (18.3) & $7(10.2)$ \\
\hline
\end{tabular}

$A D A$ adalimumab, $A E$ adverse event, $E$ event, $E O W$ every other week, $P Y$ patient-year, $U C$ ulcerative colitis

a One pancreatic carcinoma and one parathyroid tumor, which was determined to be benign but was conservatively classified as a malignancy

b One Mycobacterium avium complex infection and one cytomegalovirus infection

Similar to data observed in other clinical studies, the presence of immunogenicity did not have a clinically significant influence on the safety of adalimumab. Through week 52, the overall rate of AEs in the AAA-positive patients $(n=5)$ was 571.4 events per 100 patient-years (compared to 547.9 events/100 patient-years in the overall adalimumab $40 \mathrm{mg}$ EOW group), and all of the AEs reported in these patients were also reported in the patients without immunogenicity (data not shown).

Mean changes from baseline in laboratory variables and vital signs were similar across treatment arms (data not shown). Analyses of laboratory parameters and vital signs did not reveal any clinically relevant safety issues.

\section{Discussion}

The purpose of the present study was to compare the efficacy and safety results of adalimumab for induction and maintenance treatment in Japanese patients with moderately to severely active UC with those of patients from similar studies conducted in Western populations [6-8]. Adalimumab $160 / 80 \mathrm{mg}$ induction treatment in patients who were unresponsive to corticosteroids and/or immunomodulatory therapy significantly improved the rate of response compared with placebo at week 8 , with a rate of response similar to that observed in the pivotal phase III ULTRA 2 trial involving Western patients [7]. The current study did not demonstrate a greater rate of remission at week 8 with either adalimumab dose compared with placebo, but the adalimumab 160/80 mg induction dosing was associated with significantly greater mucosal healing at week 8 compared with placebo. Maintenance therapy with adalimumab $40 \mathrm{mg}$ EOW was associated with increasing rates of remission over time, with statistical separation compared with placebo observed at weeks 32 and 52. Rates of response and mucosal healing during maintenance treatment were also greater with adalimumab compared with placebo. Maintenance therapy was accompanied by greater rates of mild or normal subscores for RBS, PGA, and stool frequency, and a higher rate of IBDQ response compared with placebo. Additionally, adalimumab therapy was associated with meaningful rates of steroid discontinuation in patients taking steroids at baseline, with approximately $48 \%$ of patients becoming steroid-free at week 52. Greater rates of steroid-free remission were also observed with adalimumab compared with placebo. 
Because UC is less common in Japan than in the West, it would have been difficult to enroll a large number of Japanese patients; thus, the current study has a similar study design to that of previously published Western studies $[6,7]$ to allow comparison for directionality of effect. Western studies have reported a significant effect of adalimumab on the induction (at week 8, 16.5-18.5\% of patients who received $160 / 80 \mathrm{mg} ; P<0.05$ vs placebo) [6, 7] and maintenance (at week 52, $17.3 \%$ of patients who received 160/80 $\mathrm{mg}$ induction followed by $40 \mathrm{mg}$ EOW; $P<0.005$ vs placebo) [7] of remission in patients with moderately to severely active UC who had failed therapy with steroids and/or immunomodulators (including some patients who had failed infliximab in one of the Western studies). Given previous data that demonstrated similar efficacy of adalimumab in Japanese and Western patients with CD [10], it was expected that the efficacy of adalimumab in patients with UC would be similar to that observed in Western patients. With the exception of week 8 remission, the results in this study were generally consistent with those observed in the Western studies [6, 7]. In the current study, a high rate of remission was noted in the placebo arm among patients taking steroids at baseline. These findings are consistent with the known short-term effects of steroids to reduce symptoms. It is possible that, unlike in the Western studies, the patients in the Japanese study who were taking steroids at baseline may not have been truly steroid-resistant. This phenomenon was not noted at week 52, which is also consistent with the known lack of long-term efficacy noted with steroid therapy [17].

Serum adalimumab concentrations at weeks 2 and 4 after induction therapy with adalimumab 160/80 were comparable in Japanese patients with UC $(\sim 13 \mu \mathrm{g} / \mathrm{mL})$ and in Western patients with UC $(\sim 12 \mu \mathrm{g} / \mathrm{mL})$ [18]. During maintenance therapy, mean serum adalimumab concentrations among patients who did not enter the rescue arm were $9.3 \mu \mathrm{g} / \mathrm{mL}$ in patients with remission at week 52 (compared with $10.8 \mu \mathrm{g} / \mathrm{mL}$ in Western patients) and $6.8 \mu \mathrm{g} / \mathrm{mL}$ in patients without remission (compared with $6.2 \mu \mathrm{g} / \mathrm{mL}$ in Western patients) [7]. As was noted in the Western study [7], there is substantial overlap in concentrations by remission status at each time point. The immunogenicity rate in this study was comparable with the Western study (5.0 and $5.3 \%$, respectively) [7], similar to observations in previous studies in Japanese patients [10, 19].

Overall, the AE profile of adalimumab was similar to that reported in Western patients with moderately to severely active UC [6, 7] and trials of adalimumab in CD $[10,15,16]$. During induction therapy (weeks 0-8), treatment with adalimumab 160/80 $\mathrm{mg}$ was associated with an overall rate of AEs similar to that observed with placebo. Maintenance therapy with adalimumab $40 \mathrm{mg}$ EOW was associated with a similar overall rate of AEs and serious AEs compared with placebo; both of these types of events were observed less frequently among patients with response to adalimumab at week 8 .

Serious infections, including tuberculosis, were observed in patients treated with adalimumab in this and other studies, and highlight the need for clinicians to carefully weigh the potential benefits and risks of adalimumab in patients when selecting a treatment. The patient who developed tuberculosis in this study had other risk factors for tuberculosis, including increased age [20] and concomitant high-dose corticosteroid use [21]. This patient had undergone standard screening for tuberculosis (purified protein derivative skin testing and chest X-ray), consistent with the recommendations in the Japanese prescribing information for adalimumab [22]. Cases of tuberculosis have been observed in adalimumab-treated patients with negative screening tests [22], and may represent new-onset infections or false-negative testing, which may be more likely in patients taking concomitant corticosteroids or immunomodulators [20]. Clinicians initiating adalimumab in patients on combination immunosuppressive therapy or patients with other risk factors for infections should carefully monitor these patients for signs and symptoms, and have a high index for suspicion of infection in patients with symptoms suggestive of tuberculosis (e.g., persistent cough, weight loss, fever).

The decision to continue long-term therapy in patients with UC is generally based on response to induction therapy. This approach is consistent with clinical practice and international expert guidelines [23]. Therefore, rates of response, remission, and mucosal healing at week 52 were evaluated in patients who achieved response per either full or partial Mayo score at week 8. Consistent with the results noted in the ULTRA 2 Western maintenance study [24], week 8 response to adalimumab in the current study was associated with greater rates of response, remission, and mucosal healing compared with the overall adalimumab population at week 52. The greater efficacy over time, coupled with a lower rate of overall AEs in week 8 responders relative to the overall adalimumab population, together support the favorable benefit/risk profile of adalimumab maintenance therapy in patients exhibiting an early response to induction therapy.

The study database allowed for comparison of remission rate determinations using different methodologies for capturing stool frequency and rectal bleeding subscores, which are based upon patient daily diary entries. There is no standard method for capturing the values for these subscores at a patient visit, and this may have implications on the rates of remission observed in clinical trials. In this trial and in the Western adalimumab UC studies, the worst daily diary entry over the 3 days prior to the patient visit was used to 
determine each subscore. Using this method, a patient who recorded stool frequency subscores of 3,0 , and 0 over the 3 days prior to the visit would be assigned a value of 3 for the Mayo stool frequency subscore. Similarly, a patient with subscores of 3,3 , and 3 on 3 consecutive days would also be assigned a score of 3 for this subscore. Based upon the subscore assigned for that study visit, these patients would be considered to be equivalent in terms of stool frequency, even though the degree of their symptoms differs. If the subscores were determined using an average of the 3 days, the first patient would be assigned a stool frequency subscore of 1 and the second patient would receive a score of 3 . This example illustrates how the utilization of worst-case methodology in scoring could have a disproportionate influence on efficacy outcomes with active therapy, particularly if the treatment benefits led to improvement, but not complete control of symptoms. In this trial, week 8 remission rates calculated based on patient diary entries for the stool frequency and rectal bleeding subscores were lowest when the worst values of 3 or 5 days were used compared to the use of average daily entries. Additionally, the reduction in remission rates using the worst rank methodology compared to the average was more pronounced in the adalimumab treatment arms than the placebo arm. The greatest remission rates and the largest treatment effect sizes occurred with the use of the average of 5 days of diary entries. We chose to analyze week 8 remission because patients were not able to escape to the rescue arm before that time, and the escape criteria were based upon Mayo scores calculated using the worst rank of 3 days of diary entries, which may have influenced later results. These findings highlight the importance of considering the effect of endpoint determination methodology during clinical trial design and interpretation of clinical trial data.

A limitation of the current study is its relatively small sample size. Additionally, this study did not evaluate the efficacy of adalimumab in Japanese patients who had previously received other biologic therapies such as infliximab.

In conclusion, adalimumab induction therapy with $160 / 80 \mathrm{mg}$ was superior to placebo in achievement of early response and mucosal healing, and maintenance therapy with $40 \mathrm{mg}$ every other week was associated with maintenance of these effects, as well as achievement of remission at week 32 and 52 in patients with inadequate response to corticosteroids and/or immunosuppressive agents. The similarity of the clinical remission rates after 52 weeks of adalimumab therapy observed in this relatively small study with those observed in large, pivotal clinical trials [6, 7] of adalimumab in Western populations supports the use of adalimumab in Japanese patients with moderately to severely active UC, particularly in patients who demonstrate response by week 8 to induction therapy.
Acknowledgments The study was designed, conducted, and funded by AbbVie Inc and Eisai Co, Ltd. AbbVie and Eisai participated in the interpretation of data, review, and approval of the manuscript. The authors would like to thank the investigators who participated in this trial: Akira Ando, Shiga University of Medical Science Hospital; Akira Tari, Hiroshima Red Cross Hospital \& Atomic-bomb Survivors Hospital; Bunei Iizuka, Tokyo Women's Medical University Hospital; Fukunori Kinjo, University of the Ryukyu; Haruhiko Ogata, Keio University School of Medicine; Hideyuki Inoue, Kagawa University Hospital; Hiroaki Ito, Kitano Hospital, The Tazuke Kofukai Medical Research Institute; Hiroaki Takeda, Yamagata University Hospital; Hiroshi Nakase, Kyoto University Hospital; Hiroyuki Okada, Okayama University Hospital; Hisamitu Hidaka, Hidaka Daityo Clinic; Hisanori Abe, Arita GI Hospital; Ichiro Hirata, Fujita Health University Hospital; Jun Kato, Okayama University Hospital; Kazuhito Sugimura, Niigata City General Hospital; Kazuichi Okazaki, Kansai Medical University Hirakata Hospital; Kazuo Otsuka, Showa University Northern Yokohama Hospital; Kazuyoshi Yamashita, Sendai Red Cross Hospital; Keiichi Mitsuyama, Kurume University Hospital; Kenji Watanabe, Osaka City University Hospital; Kiyonori Kobayashi, Kitasato University East Hospital; Koji Yakabi, Saitama Medical Center; Kunihiko Aoyagi, Fukuoka University Hospital; Mari Mizuno, Aichi Medical University Hospital; Masaaki Sumioka, Hiroshima Prefectural Hospital; Masakazu Nagahori, Tokyo Medical and Dental University Hospital Faculty of Medicine; Masakazu Takazoe, Social Insurance Central General Hospital; Mayumi Kodama, Miyazaki Medical Center Hospital; Mikihiro Fujiya, Asahikawa Medical College Hospital; Mitsuyuki Murano, Osaka Medical College Hospital; Morio Takahashi, Koshigaya Hospital of Dokkyo Medical University; Osamu Zaha, Chibana Clinic; Ryota Hokari, National Defense Medical College Hospital; Ryouichi Suzuki, Kannai-Suzuki Clinic; Sakiko Hiraoka, Okayama University Hospital; Satoshi Tanida, Nagoya City University Hospital; Seiji Arihiro, Jikei University; Shinji Katushima, National Hospital Organization Kyoto Medical Center; Shinji Tanaka, Hiroshima University Hospital; Shiro Nakamura, The Hospital of Hyogo College of Medicine; Shusaku Yoshikawa, Kenseikai Dongo Hospital; Syunji Ishihara, Shimane University Hospital; Tadashi Yokoyama, Yokoyama Gastrointestinal Division Hospital; Takafumi Ando, Nagoya University Hospital; Takashi Kagaya, Kanazawa University Hospital; Takashi Sekikawa, Showa University Toyosu Hospital; Takayuki Matsumoto, Kyusyu University Hospital; Taro Osada, Juntendo University Hospital; Tatsuro Katsuno, Chiba University Hospital; Tetsuji Takayama, Tokushima University Hospital; Toshifumi Ashida, Sapporo Higashi Tokushukai Hospital; Toshifumi Ohkusa, The Jikei University School of Medicine Kashiwa Hospital; Toshihiro Kusaka, Kyoto Katsura Hospital; Toshiki Ichimori, Susaki Kuroshio Hospital; Toshimi Chiba, Iwate Medical University Hospital; Toshio Sakiyama, Kagoshima University Hospital; Toshiro Sugiyama, Toyama University Hospital; Toshiyuki Matsui, Fukuoka University Chikushi Hospital; Yasumi Araki, Kurume Coloproctology Center; Yoshitaka Kinouchi, Tohoku University Hospital; You Ishiguro, Hirosaki University School of Medical \& Hospital; Yuji Sakai, Matsuyama Red Cross Hospital; Yuusuke Okuyama, Japanese Red Cross Kyoto Daiichi Hospital. The authors thank Morio Ozawa, AbbVie GK, for assistance in the preparation of this manuscript. Assistance with medical writing and editing services was provided under the direction of the authors by Jeffrey Stumpf, Ph.D. and Gina Uhlenbrauck, MedThink SciCom, Inc. The financial support for these services was provided by AbbVie.

Conflict of interest Yasuo Suzuki has received fees for lectures from AbbVie GK, Eisai Co, Ltd, Kyorin Pharmaceutical Co, Ltd, and Mitsubishi Tanabe Pharma Corporation. Satoshi Motoya has received or is pending receipt of grant support from Janssen Pharmaceutical KK and Ajinomoto Pharmaceuticals Co, Ltd, and has received fees for lectures from Mitsubishi Tanabe Pharma Corporation. Hiroyuki 
Hanai has received fees for lectures from AbbVie GK, Eisai Co, Ltd, Kyorin Pharmaceutical Co, Ltd, and Mitsubishi Tanabe Pharma Corporation. Takayuki Matsumoto has no conflicts of interest to declare. Toshifumi Hibi has received or is pending receipt of grants from AbbVie, Ajinomoto Pharmaceuticals Co, Ltd, Asahi Kasei Kuraray Medical Co, Ltd, AstraZeneca Pharmaceuticals, Janssen Pharmaceutical KK, JIMRO Co, Ltd, Kyorin Pharmaceutical Co, Ltd, Otsuka Pharmaceutical Co, Ltd, Tanabe Mitsubishi Seiyaku, UCB Japan Co, Ltd, UMN Pharma Inc, and Zeria Pharmaceutical Co, Ltd, and has received fees for lectures from AbbVie, Asahi Kasei Kuraray Medical Co, Ltd, JIMRO Co, Ltd, Kyorin Pharmaceutical Co, Ltd, Tanabe Mitsubishi Seiyaku, and Zeria Pharmaceutical Co, Ltd. Anne M. Robinson, Nael M. Mostafa, Jingdong Chao, Vipin Arora, Anne Camez, and Roopal B. Thakkar are employees of AbbVie Inc and may hold AbbVie stock or options. Mamoru Watanabe has received or is pending receipt of grants from AbbVie GK, Astellas Pharma Inc, Asahi Kasei Kuraray Medical Co, Ltd, Ajinomoto Pharmaceuticals Co, Ltd, Chugai Pharmaceutical Co, Ltd, Daiichi Sankyo Co, Ltd, Eisai Co, Ltd, Kyowa Hakko Kirin Co, Ltd, Kyorin Pharmaceutical Co, Ltd, JIMRO Co, Ltd, Mitsubishi Tanabe Pharma Corporation, MSD KK, Otsuka Pharmaceutical Co, Ltd, Takeda Pharmaceutical Co, Ltd, UCB Japan Co, Ltd, and Zeria Pharmaceutical Co, Ltd, and has received payment for lectures from AbbVie GK, Eisai Co, Ltd, Kyorin Pharmaceutical Co, Ltd, and Mitsubishi Tanabe Pharma Corporation.

Open Access This article is distributed under the terms of the Creative Commons Attribution Noncommercial License which permits any noncommercial use, distribution, and reproduction in any medium, provided the original author(s) and the source are credited.

\section{References}

1. Podolsky DK. Inflammatory bowel disease. $\mathrm{N}$ Engl $\mathrm{J}$ Med. 2002;347:417-29.

2. Sands BE, Kaplan GG. The role of TNF $\alpha$ in ulcerative colitis. J Clin Pharmacol. 2007;47:930-41.

3. Hamilton MJ, Snapper SB, Blumberg RS. Update on biologic pathways in inflammatory bowel disease and their therapeutic relevance. J Gastroenterol. 2012;47:1-8.

4. Arimura Y, Isshiki H, Onodera K, et al. Characteristics of Japanese inflammatory bowel disease susceptibility loci. J Gastroenterol. 2013 (Epub ahead of print).

5. Kuwahara E, Asakura K, Nishiwaki Y, et al. Effects of family history on inflammatory bowel disease characteristics in Japanese patients. J Gastroenterol. 2012;47:961-8.

6. Reinisch W, Sandborn WJ, Hommes DW, et al. Adalimumab for induction of clinical remission in moderately to severely active ulcerative colitis: results of a randomised controlled trial. Gut. 2011;60:780-7.

7. Sandborn WJ, van Assche G, Reinisch W, et al. Adalimumab induces and maintains clinical remission in patients with moderate-to-severe ulcerative colitis. Gastroenterology. 2012;142(257-65):e3.

8. Reinisch W, Sandborn WJ, Panaccione R, et al. 52-week efficacy of adalimumab in patients with moderately to severely active ulcerative colitis who failed corticosteroids and/or immunosuppressants. Inflamm Bowel Dis. 2013;19:1700-9.

9. Asakura K, Nishiwaki Y, Inoue N, et al. Prevalence of ulcerative colitis and Crohn's disease in Japan. J Gastroenterol. 2009;44:659-65.

10. Watanabe M, Hibi T, Lomax KG, et al. Adalimumab for the induction and maintenance of clinical remission in Japanese patients with Crohn's disease. J Crohns Colitis. 2012;6:160-73.

11. Schroeder KW, Tremaine WJ, Ilstrup DM. Coated oral 5-aminosalicylic acid therapy for mildly to moderately active ulcerative colitis. A randomized study. $N$ Engl J Med. 1987;317:1625-9.

12. Guyatt G, Mitchell A, Irvine EJ, et al. A new measure of health status for clinical trials in inflammatory bowel disease. Gastroenterology. 1989;96:804-10.

13. Sandborn WJ, Feagan BG, Marano C, et al. Subcutaneous golimumab maintains clinical response in patients with moderate-tosevere ulcerative colitis. Gastroenterology. 2013 (Epub ahead of print).

14. Weisman MH, Moreland LW, Furst DE, et al. Efficacy, pharmacokinetic, and safety assessment of adalimumab, a fully human anti-tumor necrosis factor-alpha monoclonal antibody, in adults with rheumatoid arthritis receiving concomitant methotrexate: a pilot study. Clin Ther. 2003;25:1700-21.

15. Colombel J-F, Sandborn WJ, Rutgeerts P, et al. Adalimumab for maintenance of clinical response and remission in patients with Crohn's disease: the CHARM trial. Gastroenterology. 2007;132:52-65.

16. Hanauer SB, Sandborn WJ, Rutgeerts P, et al. Human anti-tumor necrosis factor monoclonal antibody (adalimumab) in Crohn's disease: the CLASSIC-I trial. Gastroenterology. 2006;130:323-33.

17. Faubion WA Jr, Loftus EV Jr, Harmsen WS, et al. The natural history of corticosteroid therapy for inflammatory bowel disease: a population-based study. Gastroenterology. 2001;121:255-60.

18. Humira [package insert]. North Chicago, IL: AbbVie Inc; 2013.

19. Miyasaka N, the CHANGE study investigators. Clinical investigation in highly disease-affected rheumatoid arthritis patients in Japan with adalimumab applying standard and general evaluation: the CHANGE study. Mod Rheumatol. 2008;18:252-62.

20. Rahier JF, Ben-Horin S, Chowers Y, et al. European evidencebased Consensus on the prevention, diagnosis and management of opportunistic infections in inflammatory bowel disease. J Crohns Colitis. 2009;3:47-91.

21. Jick SS, Lieberman ES, Rahman MU, Choi HK. Glucocorticoid use, other associated factors, and the risk of tuberculosis. Arthr Rheum. 2006;55:19-26.

22. Humira [Japanese package insert]. Tokyo, Japan: AbbVie GK and Eisai Co, Ltd; 2013.

23. Dignass A, Lindsay JO, Sturm A, et al. Second European evidence-based consensus on the diagnosis and management of ulcerative colitis part 2: current management. J Crohns Colitis. 2012;6:991-1030.

24. Sandborn WJ, Colombel J-F, D'Haens G, et al. One-year maintenance outcomes among patients with moderately-to-severely active ulcerative colitis who responded to induction therapy with adalimumab: subgroup analyses from ULTRA 2. Aliment Pharmacol Ther. 2013;37:204-13. 